\title{
AHV und Arzt
}

\section{Helfenstein}

Die AHV begleitet den Arzt praktisch während des ganzen Lebens. Die Beitragspflicht beginnt mit dem vollendeten siebzehnten Lebensjahr und endet, wenn das ordentliche Rentenalter erreicht ist. In bestimmten Fällen müssen auch nach dem Erreichen des Rentenalters AHV-Beiträge bezahlt werden. Ich versuche hier aufzuzeigen, welche AHV-Sätze angewendet und auf welcher Basis diese erhoben werden.

\section{AHV und Studienzeit}

Studierende ohne Erwerbstätigkeit mit Sitz in der Schweiz sind ab 1. Januar nach Vollendung des 20. Alterjahres beitragspflichtig. Die Abgabe beträgt Fr. 425.- pro Jahr und ist direkt an die Lehranstalt oder an die Ausgleichskasse am Sitz der Lehranstalt zu entrichten. Die Ausgleichskassen erheben zusätzlich auf den jährlich geschuldeten Betrag einen Verwaltungskostenzuschlag von etwa $3 \%$. Keine Beiträge sind geschuldet, wenn folgende Punkte zutreffen:

- Es liegt ein mit der Ausgleichskasse abgerechnetes Erwerbseinkommen aus unselbständiger Erwerbstätigkeit vor, und zwar von etwa Fr. 3000.- Bruttolohn (pro Jahr). Kleinere Einkommen werden am Minimalbetrag angerechnet. $\mathrm{Zu}$ beachten ist in diesem Fall, dass die Beitragspflicht bereits im Jahr beginnt, wo das achtzehnte Lebensjahr erreicht wird.

- Der Arzt ist verheiratet und der andere Ehegatte rechnet mit der Ausgleichskasse mindestens Fr. 850.- (doppelter Minimalbetrag) ab.

\section{AHV und unselbständige Erwerbstätigkeit (Lohnempfänger)}

Alle Arbeitnehmer ab Jahrgang 1985 sind abgabepflichtig. Die Beiträge müssen jedoch nicht selber einbezahlt werden, sondern werden den Angestellten vom Bruttolohn in Abzug gebracht. Der Arbeitgeber ist verpflichtet, diese Beträge und auch die Arbeitgeberbeiträge mit der Ausgleichskasse abzurechnen. Damit später bei der Pensionierung keine Kürzungen erfolgen, sind
Beitragslücken unbedingt zu vermeiden. Somit muss bei jedem Stellenwechsel zwingend die graue persönliche AHV-Karte dem Arbeitgeber ausgehändigt werden. Der Arbeitgeber leitet diese Karte an seine Ausgleichskasse weiter. Diese nimmt die entsprechenden Einträge vor und schickt die Karte retour. Die Beiträge setzen sich wie folgt zusammen:

\begin{tabular}{lr} 
AHV & $8,4 \%$ \\
\hline IV & $1,4 \%$ \\
\hline EO & $0,3 \%$ \\
\hline ALV & $2,5 \%$ \\
\hline Total & $12,6 \%$
\end{tabular}

Von diesem Prozentsatz tragen Arbeitnehmer und Arbeitgeber je die Hälfte, und zwar 6,3\%. Was alles zum Bruttolohn gehört, kann dem Merkblatt Nr. 2.01 entnommen werden. Dieses Merkblatt kann bei den kantonalen Ausgleichskassen bezogen werden. Ärzte, die eigenes Personal beschäftigen, müssen damit rechnen, dass sie von Zeit zu Zeit von der AHV kontrolliert werden. Damit bei diesen Kontrollen keine bösen Überraschungen entstehen, sind rechtzeitig offene Lohnfragen mit der AHV oder dem Berater zu klären. Hier die häufigsten durch Kontrollen beanstandeten Fehler:

- fehlende Lohnbestandteile (Naturallöhne, Reinigungslöhne, Spesen mit Lohncharakter);

- falsche Berechnungen des massgebenden Lohnes;

- nicht erfasste Arbeitnehmer (Aushilfen, Lehrlinge, Rentner).

Werden in einer Arztpraxis Rentner beschäftigt, oder arbeitet der Arzt nach dem Erreichen des ordentlichen Rentenalters im Angestelltenverhältnis weiter, dann muss nicht der ganze Bruttolohn mit der AHV abgerechnet werden. Es gilt in diesen Fällen ein Freibetrag von Fr. 16800.- pro Jahr oder Fr. 1400.- pro Monat. Die Rente kann damit jedoch nicht mehr aufgebessert werden. Die den Freibetrag übersteigenden Löhne sind abgabepflichtig und sind als zusätzliche Steuer zu verstehen. 


\section{AHV und selbständige Erwerbstätigkeit}

Die Ausgleichskassen klären ab, welchen Status für den Arzt zutrifft. Als selbständigerwerbend gilt, wer unter eigenem Namen und auf eigene Rechnung arbeitet und sich in unabhängiger Stellung befindet. Zudem trägt der Arzt das eigene wirtschaftliche Risiko selber. In der Praxis trifft dies meistens $\mathrm{zu}$, wenn der Arzt eine eigene Praxis eröffnet und diese als Einzelfirma oder Praxisgemeinschaft führt. Die Höhe der Beiträge setzt sich wie folgt fest:

\begin{tabular}{ll} 
AHV & $7,8 \%$ \\
\hline IV & $1,4 \%$ \\
\hline EO & $0,3 \%$ \\
\hline Total & $9,5 \%$
\end{tabular}

Dieser Satz kommt zur Anwendung, sofern mindestens ein Jahreseinkommen von Fr. 50700.erzielt wird. Bei tieferen Jahreseinkommen ab Fr. 8500.- kommen abgestufte Sätze zum Zug. Auch hier gilt ein jährlicher Minimalbetrag von Fr. 425.-, der zu entrichten ist, obwohl beim Aufbau einer Arztpraxis in der Regel sogar Verluste erzielt werden. Zudem können die Verluste nicht mit späteren Einkommen verrechnet werden. Jährliche AHV-Einkommen, die Fr. 75 960.- übersteigen, sind nicht mehr rentenbildend und stellen eine zusätzliche Steuer dar.

Die Veranlagungsbehörde für die direkte Bundessteuer meldet das AHV-pflichtige Einkommen an die Ausgleichskasse des Arztes. Dabei ist nicht das steuerbare Einkommen wichtig, sondern das Einkommen aus der Praxistätigkeit (Gewinn). Zu diesem Gewinn werden die persönlichen AHV-Beiträge des Arztes addiert. Von diesem Betrag wird noch ein Zins auf das
Eigenkapital in der Praxis berechnet und abgezogen.

Bevor der Arzt die genauen Zahlen kennt, zahlt er für seine persönlichen AHV-Beiträge Akontozahlungen. Besteht die Arztpraxis noch nicht lange, so meldet der Inhaber telefonisch sein mutmassliches AHV-Einkommen an die Ausgleichskasse (Medisuisse in St. Gallen). Probleme entstehen dann, wenn die persönliche Steuererklärung mit grosser Verspätung eingereicht wird, die Einschätzungsbehörde lange mit der Veranlagung zuwartet und der Arzt Einkommensschwankungen in der eigenen Arztpraxis hat. Es kann vorkommen, dass die geleisteten Akontozahlungen während mehrerer Jahre zu tief waren und eine grosse Nachzahlung fällig wird. Dazu kommen noch je nach Höhe der Differenz und des Zahlungsdatums Verzugszinsen (5\%). Für zuviel bezahlte Akontobeiträge wird ein Vergütungszins gutgeschrieben.

\section{Fazit}

$\mathrm{Zu}$ jeder Abschlussgestaltung gehört auch der Miteinbezug der AHV-Faktoren. Können AHVBeitragsdifferenzen rechtzeitig nachgemeldet werden, spart der Arzt immerhin die Verzugszinsen. Auf dem Praxisergebnis können für die ausstehenden AHV-Beiträge Rückstellungen gebildet werden. Gerade bei neuen Arztpraxen mit einem sehr tiefen Einkommen muss unbedingt beachtet werden, dass bei einem Schadenfall (Invalidität) nicht die maximale Rente erwartet werden darf. Allfällige Lücken müssen privat abgedeckt werden. Als Lösungsansatz kann auch das Verschieben von Abschreibungen in Jahre mit besseren Einkommen betrachtet werden. 


\section{FMH SERVICES}

\section{Unsere Treuhandspezialisten}

Geschäftsstelle Muri b. Bern, Herr Peter Schneider, Thorackerstrasse 3, 3074 Muri bei Bern, Tel. 0319518840 . Fax 0319518839 - E-Mail: peter.schneider@fmhtreuhand.ch

Geschäftsstelle Ostermundigen, Herr Harry Huwiler, Bernstrasse 102, 3072 Ostermundigen 2, Tel. 0319390139 - Fax 0319390131 - E-Mail: harry.huwiler@fmhtreuhand.ch

Geschäftsstelle Olten, Herr Peter Senn, Baslerstrasse 32, 4603 Olten,

Tel. 0622059035 . Fax 0622059039 - E-Mail: peter.senn@fmhtreuhand.ch

Geschäftsstelle Basel, Herr Linus Cavegn, Hirzbodenweg 103, 4020 Basel,

Tel. 0613195121 . Fax 0613195252 . E-Mail: linus.cavegn@fmhtreuhand.ch

Geschäftsstelle Lohn-Ammannsegg SO, Herr Rolf Lehmann, Alte Bernstr. 53, 4573 Lohn-Ammannsegg, Tel. 0326775442 . Fax 0326775441 . E-Mail: rolf.lehmann@fmhtreuhand.ch

Geschäftsstelle Muri / AG, Herr Roland Bütler, Kirchenfeldstrasse 6, 5630 Muri, Tel. 0566640309 • Fax 0566645566 • E-Mail: roland.buetler@fmhtreuhand.ch

Geschäftsstelle Cham, Herr Guido Schmid, Alte Steinhauserstrasse 1, 6330 Cham, Tel. 0417486290 . Fax 0417486201 . E-Mail: guido.schmid@fmhtreuhand.ch

Geschäftsstelle Sursee, Herr Patrik Dahinden, Christoph-Schnyder-Strasse 46, 6210 Sursee, Tel. 0419267045 . Fax 0419267025 . E-Mail: patrik.dahinden@fmhtreuhand.ch

Geschäftsstelle Stans, Herr Marcel Helfenstein, Hansmatt 32, 6370 Stans,

Tel. 0416111821 • Fax 0416111820 . E-Mail: marcel.helfenstein@fmhtreuhand.ch

Geschäftsstelle Zürich-Wiedikon, Herr Christoph Lautenschlager, Steinstrasse 21, 8036 Zürich, Tel. 014571575 . Fax 014571516 . E-Mail: christoph.lautenschlager@fmhtreuhand.ch

Geschäftsstelle Winterthur, Herr Urs Gross, Ackeretstrasse 3, 8407 Winterthur, Tel. 0522240241 . Fax 0522223328 . E-Mail: urs.gross@fmhtreuhand.ch

Geschäftsstelle Weinfelden, Herr Adrian Hartmann, Marktplatz 6, 8570 Weinfelden, Tel. 0716228686 . Fax 0716228688 . E-Mail: adrian.hartmann@fmhtreuhand.ch

Geschäftsstelle Oberuzwil / SG, Herr Martin Brenner, Wiesentalstr. 22, 9242 Oberuzwil, Tel. 0719513066 . Fax 0719514771 . E-Mail: martin.brenner@fmhtreuhand.ch

Geschäftsstelle Speicher / St. Gallen, Herr Jürg Schmid, Zaun 55, 9042 Speicher, Tel. 0713442175 . Fax 0713443980 . E-Mail: juerg.schmid@fmhtreuhand.ch

Geschäftsstelle Au / SG, Herr Anibal Alghisi, Berneckerstrasse 9, 9434 Au, Tel. 0717401787 . Fax 0717401785 . E-Mail: anibal.alghisi@fmhtreuhand.ch

Geschäftsstelle Chur, p.Adr. Riedi Ruffner Theus AG, Poststrasse 22, 7002 Chur, Tel. 0812584646 - Fax 0812584647 . E-Mail: giorgio.cappellin@fmhtreuhand.ch

Geschäftsstelle Lausanne, Monsieur Michel Favre, Rue Pichard 20, 1003 Lausanne, Tel. 0213175454 . Fax 0213175453 . E-Mail: fiduciaire.favre@fmhfiduciaire.ch

Geschäftsstelle Genf, Monsieur Jean-Charles Terraz, rue Pedro-Meylan 4, 1208 Genf, Tel. 0227866870 • Fax 0227866659 . E-Mail: jeancharles.terraz@fmhfiduciaire.ch

Geschäftsstelle Sierre, Monsieur Jean-Pierre Chevalley, Avenue des Alpes 6, 3960 Sierre, Tel. 0274551675 . Fax 0274550644 . E-Mail: jeanpierre.chevalley@fmhfiduciaire.ch

Geschäftsstelle Martigny, Monsieur Gérald Claude, av. des Prés-Beudin 20, 1920 Martigny, Tel. 0277232009 . Fax 0277232001 . E-Mail: gerald.claude@fmhfiduciaire.ch

Geschäftsstelle Chiasso, Signor Franco Pozzi, Corso San Gottardo 89, 6830 Chiasso, Tel. 0916976080 . Fax 0916836826 . E-Mail: franco.pozzi@fmhfiduciaria.ch 